\title{
X-shooter search for outgassing from main belt comet P/2012 T1 (Pan-STARRS) ${ }^{\star}$
}

\author{
C. Snodgrass ${ }^{1}$, B. Yang ${ }^{2}$, and A. Fitzsimmons ${ }^{3}$ \\ 1 Planetary and Space Sciences, School of Physical Sciences, The Open University, Milton Keynes, MK7 6AA, UK \\ e-mail: Colin.Snodgrass@open.ac.uk \\ 2 European Southern Observatory, Alonso de Cordova 3107, Vitacura, Casilla 19001, Santiago, Chile \\ 3 Astrophysics Research Centre, School of Mathematics and Physics, Queen's University Belfast, Belfast, BT7 1NN, UK
}

Received 28 April 2017 / Accepted 17 June 2017

\begin{abstract}
Context. Main belt comets are a recently identified population of minor bodies with stable asteroid-like orbits but cometary appearances. Sublimation of water ice is the most likely mechanism for their recurrent activity (i.e. dust tails and dust comae), although there has been no direct detection of gas. These peculiar objects could hold the key to the origin of water on Earth.

Aims. In this paper we present a search for the gas responsible for lifting dust from P/2012 T1 (Pan-STARRS), and review previous attempts at such measurements. To date such searches have mainly been indirect, looking for the common cometary gas $\mathrm{CN}$ rather than gasses related to water itself.

Methods. We use the VLT and X-shooter to search for emission from $\mathrm{OH}$ in the UV, a direct dissociation product of water.

Results. We do not detect any emission lines, and place an upper limit on water production rate from $\mathrm{P} / 2012 \mathrm{~T} 1$ of $8-9 \times$ $10^{25}$ molecules s ${ }^{-1}$. This is similar to limits derived from observations using the Herschel space telescope.

Conclusions. We conclude that the best current facilities are incapable of detecting water emission at the exceptionally low levels required to produce the observed activity in main belt comets.
\end{abstract}

Key words. comets: individual: P/2012 T1 (Pan-STARRS) - techniques: spectroscopic

\section{Introduction}

P/2012 T1 (Pan-STARRS) was discovered in October 2012 and immediately recognised as a potential new member of the recently identified class of main belt comets (MBCs, Hsieh \& Jewitt 2006). It was the tenth candidate found and the third found by the Pan-STARRS survey (Tholen et al. 2012). MBCs present a puzzle - they have stable asteroid-like orbits, yet have comet-like appearances, implying the presence of volatile ices in their nuclei despite existing in a thermal environment that rules out surface ice. Although dust release can be caused by purely asteroidal processes - e.g. collisions, rotational breakup, and/or binary merger (Snodgrass et al. 2010; Stevenson et al. 2012; Jewitt et al. 2013; Hainaut et al. 2014) - there is indirect evidence that some MBCs are genuine comets, with activity driven by sublimation of ice. The most convincing argument is the repeated activity seen in objects such as 133P/Elst-Pizarro and 238P/Read (Hsieh et al. 2010, 2011), which is very difficult to explain by any other mechanism. Newer candidates, such as $\mathrm{P} / 2012 \mathrm{~T}$, have not been known long enough to see if they repeat their activity each orbit, but morphological studies can be used to test whether a dust tail is due to prolonged activity or single events (like collisions). Moreno et al. (2013) find that the morphology of $\mathrm{P} / 2012 \mathrm{~T} 1$ is best explained by comet activity lasting a few months. However, there has not yet been a conclusive proof that MBCs are sublimation driven, which would be provided by a direct detection of gas in the coma of one of these comets.

\footnotetext{
$\star$ Based on observations collected at the European Organisation for Astronomical Research in the Southern Hemisphere under ESO programme 290.C-5007(A).
}

In this paper we review previous attempts to detect outgassing from MBCs, before presenting our own VLT/X-shooter observations of $\mathrm{P} / 2012 \mathrm{~T} 1$. We then discuss the implications of the non-detections, and compare this with expected activity levels.

\section{Searches for outgassing from MBCs}

Spectroscopy reveals the gaseous species in cometary comae via various strong fluorescence emission bands, mostly excited by solar UV radiation. In very bright comets it is possible to use high-resolution spectroscopy, and/or a wide range of wavelengths (ultraviolet (UV), visible light, infrared, sub-mm and radio), to get a very detailed measurement of the contents of the coma, even at an isotopic level (e.g. Bockelée-Morvan et al. 2004). From these, we infer the presence of volatile ices (known as the parent species) in the nucleus. As MBCs are very faint comets, there is little chance to detect any but the strongest emission lines. These are found in the UV/visible range; $\mathrm{OH}$ at $308 \mathrm{~nm}$ and $\mathrm{CN}$ at $389 \mathrm{~nm}$ are the easiest to detect in most comets. The $\mathrm{OH}$ band is the stronger of the two, but it is more difficult to observe from the ground due to absorption by ozone in the terrestrial atmosphere. Therefore $\mathrm{CN}$ is the species of choice for detecting outgassing from a faint comet, and this has been tried with a number of MBCs (Table 1). None of these attempts were successful, despite using some of the world's largest telescopes, but allow upper limits on the gas production to be made. By assuming a "typical" $\mathrm{CN}: \mathrm{H}_{2} \mathrm{O}$ ratio for a comet, the limit on $\mathrm{CN}$ production is converted into a limit in the water production. The limits found all correspond to water production rates $Q\left(\mathrm{H}_{2} \mathrm{O}\right) \leq 10^{26}$ molecules per second (see Table 1 ). 
Table 1. Previous upper limits on MBC gas production.

\begin{tabular}{llccccl}
\hline \hline MBC & Telescope & $\begin{array}{c}r \\
(\mathrm{AU})\end{array}$ & Obs. date & $\begin{array}{c}Q(\mathrm{CN}) \\
\text { molec. } \mathrm{s}^{-1}\end{array}$ & $\begin{array}{c}Q\left(\mathrm{H}_{2} \mathrm{O}\right) \\
\text { molec. }^{-1}\end{array}$ & Reference \\
\hline 133P & VLT & 2.64 & $2007 / 07 / 21$ & $1.3 \times 10^{21}$ & $1.5 \times 10^{24}$ & Licandro et al. (2011) \\
176P & Herschel & 2.58 & $2011 / 08 / 08$ & - & $4 \times 10^{25}$ & de Val-Borro et al. (2012) \\
324P & Keck & 2.66 & $2010 / 10 / 05$ & $3 \times 10^{23}$ & $1 \times 10^{26}$ & Hsieh et al. (2012c) \\
259P & Keck & 1.86 & $2008 / 09 / 30$ & $1.4 \times 10^{23}$ & $5 \times 10^{25}$ & Jewitt et al. (2009) \\
288P & Gemini & 2.52 & $2011 / 12 / 02$ & $1.3 \times 10^{24}$ & $1 \times 10^{26}$ & Hsieh et al. (2012b) \\
& GTC & 2.52 & $2011 / 11 / 29$ & $1.1 \times 10^{24}$ & - & Licandro et al. (2013) \\
596 & Keck & 3.1 & $2010 / 12 / 17$ & $9 \times 10^{23}$ & $1 \times 10^{27}$ & Hsieh et al. (2012a) \\
P/2013 R3 & Keck & 2.23 & $2013 / 10 / 01$ & $1.2 \times 10^{23}$ & $4.3 \times 10^{25}$ & Jewitt et al. (2014) \\
313P & Keck & 2.41 & $2014 / 10 / 22$ & $1.8 \times 10^{23}$ & $6 \times 10^{25}$ & Jewitt et al. (2015) \\
P/2012 T1 & Keck & 2.42 & $2012 / 10 / 19$ & $1.5 \times 10^{23}$ & $5 \times 10^{25}$ & Hsieh et al. (2013) \\
& Herschel & 2.50 & $2013 / 01 / 16$ & - & $7.6 \times 10^{25}$ & O'Rourke et al. (2013) \\
\hline
\end{tabular}

Notes. ${ }^{(*)} 176 \mathrm{P}$ was not visibly active (no dust release) at the time of the Herschel observations. ${ }^{(\dagger)}$ The dust ejected from (596) Scheila was almost certainly due to a collision, rather than cometary activity (e.g. Bodewits et al. 2011; Ishiguro et al. 2011; Yang \& Hsieh 2011).

This value would be quite low for a Jupiter family comet close to perihelion, but is less constraining at heliocentric distances $r \approx 3 \mathrm{AU}$, where much lower activity levels are expected. Searches for $\mathrm{CN}$ have reached the sensitivity limit of current large telescopes, but do not rule out sublimation as the cause of the very low activity levels seen in MBCs - these non-detections are not evidence for a lack of ice (Jewitt 2012).

Moreover, theoretical work suggests that only water ice can survive in an MBC, and more volatile ices (such as $\mathrm{HCN}$, which is thought to be the parent species for $\mathrm{CN}$ ) would have been lost. Thermal models which calculate the heat conduction into the interior of asteroids find that water ice can survive in MBClike orbits when buried at depths of around $100 \mathrm{~m}$, but that the entire interior of a $\mathrm{km}$-scale body will reach a temperature above the HCN sublimation point (Prialnik \& Rosenberg 2009; Capria et al. 2012). While it is possible to imagine that more volatile species survive as gas trapped within water ice, they certainly cannot be retained in large amounts, and will definitely not have the same relative abundance as other comets.

This means that the assumption of a similar $\mathrm{CN}: \mathrm{H}_{2} \mathrm{O}$ ratio for $\mathrm{MBCs}$ is probably incorrect, and searches for $\mathrm{CN}$ gas, as an indicator of ice sublimation, may never succeed, no matter how sensitive. Instead the search must focus on direct detection of water or its dissociation products (e.g. OH). Two attempts have been made using the Herschel space telescope to look for water directly (176P - de Val-Borro et al. 2012; P/2012 T1 - O'Rourke et al. 2013), but both provided only upper limits (Table 1). In the first case the observers were also unlucky that $176 \mathrm{P}$ did not return to activity when predicted - no dust was seen in visible wavelength images taken at around the same time as the Herschel observations (Hsieh et al. 2014). For P/2012 T1 there was clearly visible dust activity at the time of the Herschel observations, but no gas was seen. This result is discussed further in Sect. 4.

\section{X-shooter observations of $\mathrm{P} / 2012 \mathrm{T1}$}

\subsection{Observations}

Our approach, rather than trying to detect water directly, is to search for the strong UV emission band from $\mathrm{OH}(0-0)$ at $308 \mathrm{~nm}$. The photodissociation of $\mathrm{H}_{2} \mathrm{O} \rightarrow \mathrm{OH}+\mathrm{H}$ in cometary comae is well understood, and can therefore be used to obtain reliable water production rates from $\mathrm{OH}$ line strengths with a
Table 2. Observations details.

\begin{tabular}{lccccc}
\hline \hline UT date & $\begin{array}{c}r \\
(\mathrm{AU})\end{array}$ & $\begin{array}{c}\Delta \\
(\mathrm{AU})\end{array}$ & $\begin{array}{c}\alpha \\
(\mathrm{deg})\end{array}$ & airm. & $N_{\text {obs }}{ }^{\dagger}$ \\
\hline $2012 / 12 / 14$ & 2.46 & 1.67 & 17 & 1.2 & 4 \\
$2012 / 12 / 18$ & 2.47 & 1.71 & 18 & $1.2-1.4$ & 6 \\
\hline
\end{tabular}

Notes. ${ }^{(\dagger)}$ Number of observations. Each consists of parallel exposures of $900 \mathrm{~s}$ duration in UVB and NIR arms, and $855 \mathrm{~s}$ in VIS.

few assumptions. The $\mathrm{OH}$ band is also the strongest in typical comet spectra (e.g. Feldman et al. 2004), and should therefore produce a clear signal in even low activity comets. The difficulty with this band is the strong absorption in the terrestrial atmosphere due to ozone at UV wavelengths, which is around $80 \%$ at $308 \mathrm{~nm}$. Good skies, large telescopes and very sensitive instrumentation are therefore required to recover the remaining photons, and many modern low-to-medium resolution spectrographs do not even try to get to such blue wavelengths (VLT/FORS, for example, cuts off below $350 \mathrm{~nm}$ by design).

$\mathrm{X}$-shooter is designed to provide medium-to-high resolution spectroscopy over a very wide wavelength range in one shot, using three arms optimised for UVB, visible and near-infrared (NIR) wavelengths (Vernet et al. 2011). Its bluest order covers $300-320 \mathrm{~nm}$, making it potentially sensitive to $\mathrm{OH}$ emission lines in comets. Combined with the collecting power of the $8 \mathrm{~m} \mathrm{VLT}$, detection of low production rate cometary outgassing should be possible with X-shooter. The very wide wavelength range (up to $2.5 \mu \mathrm{m}$ ) also gives another advantage of $\mathrm{X}$-shooter for studying comets - many other potential emission lines can also be investigated simultaneously, including $\mathrm{CN}$ (even if is not expected in $\mathrm{MBCs}$ ), and the continuum from the dust can give clues about its composition. The NIR arm covers the range where solid state absorption from ice grains in the coma could potentially be detected, at 1.5 and $2.0 \mu \mathrm{m}$. For example, Snodgrass et al. (2016) used X-shooter to observe 67P/Churyumov-Gerasimenko around the time of the Philae landing, although the low activity level of the comet at the time (at $r=3 \mathrm{AU}$ ) and poor visibility (airmass $\sim 2$ ) meant that only upper limits on gas production rates could be measured.

Following the discovery of $\mathrm{P} / 2012 \mathrm{~T} 1$ and initial visible wavelength photometry to assess its total brightness and dust production, we applied for and were awarded four hours of 


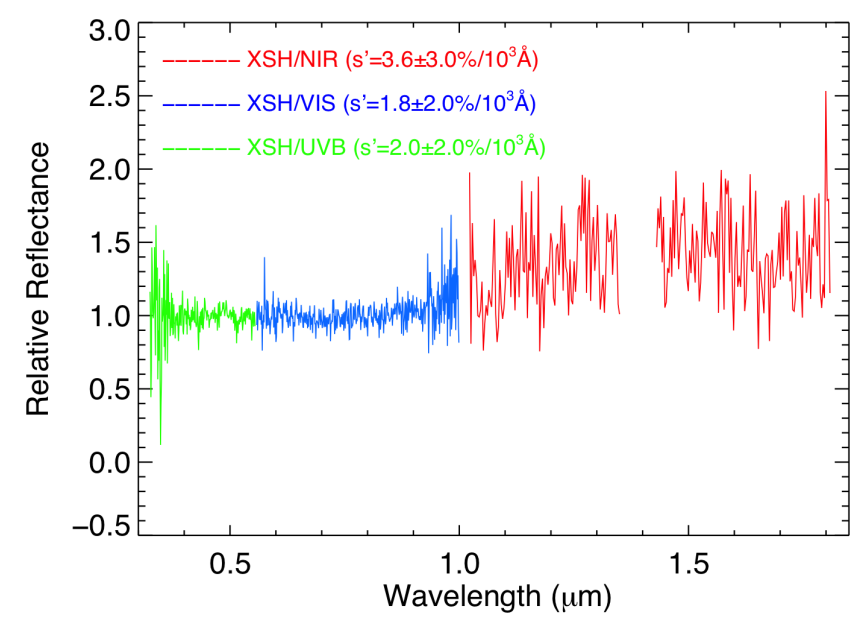

Fig. 1. Combined $X$-shooter reflectance spectrum of $P / 2012 \mathrm{~T} 1$. The UVB and VIS spectra have been binned into intervals of $10 \AA$ and the NIR spectrum has been binned into intervals of $60 \AA$ to increase the signal-to-noise. The two spectral regions, centred at 1.4 and $1.9 \mu \mathrm{m}$, that are heavily contaminated by the telluric absorptions have been cut out.

Director's discretionary time to try these challenging X-shooter observations. The observations took place in service mode during dark time in December 2012, within three months of the initial discovery. Details on the observing geometry are given in Table 2. We used slit widths 1.0, 0.9 and 0.9 arcsec in the UVB, VIS and NIR arms, respectively. We also observed the solar analogue star SA93-101 immediately after the comet on both nights.

\subsection{Data reduction}

We first processed the data using the X-shooter pipeline (Modigliani et al. 2010), which performed the following steps: bias-subtraction, cosmic ray detection and removal, flat-fielding, wavelength-calibration and order-merging. Our target was rather faint, especially in the bluest orders of the UVB arm, where the signal level was very low. In order to get reliable signal, we extracted the 1D spectrum from a two-dimensional image using the apall procedure from IRAF. We only applied flux calibration to the UVB section, where the important $\mathrm{OH}$ and $\mathrm{CN}$ emission lines are located. We removed telluric absorption features as well as the solar gradient via dividing the comet spectrum by that of the solar analogue star, SA93-101. The resulting reflectance spectrum is shown in Fig. 1.

\subsection{Dust continuum}

The reflectance spectrum has a remarkably flat slope, in agreement with the Keck spectrum published by Hsieh et al. (2013), and in contrast to the red slopes typically seen in most comets (e.g. Hadamcik \& Levasseur-Regourd 2009). The neutral slope of the dust spectrum matches the nucleus spectrum measured for other MBCs (Licandro et al. 2011), assuming that the coma and nucleus spectra can be compared. Observations at $67 \mathrm{P}$ showed that the total coma spectrum matched the nucleus (Snodgrass et al. 2016), but the dust size distribution and grain surface composition can influence coma reflectance properties (e.g. Kolokolova et al. 2004). The comet spectrum appears rather weak in the NIR, especially so in the $K$-band. We therefore present the NIR portion of the continuum in the $J$ and $H$ band only. Although the dust slope appears slightly redder at wavelengths $>1 \mu \mathrm{m}(4 \pm 3 \% / 1000 \AA)$, it is consistent, within the

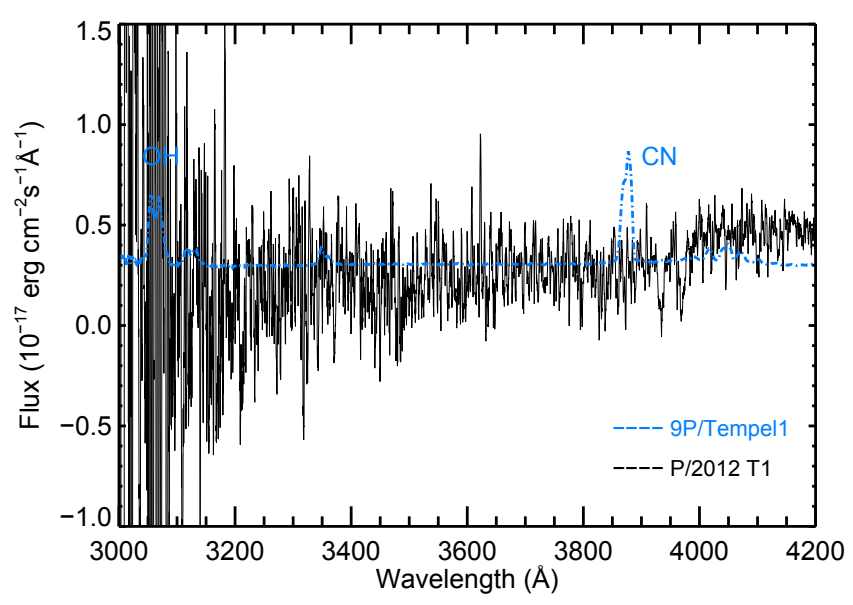

Fig. 2. Combined flux spectrum of $\mathrm{P} / 2012 \mathrm{~T} 1$ (without any solar spectrum removal), zoomed in on the UV region around the expected $\mathrm{OH}$ and $\mathrm{CN}$ emission bands, which has been smoothed over 20 adjacent points (about $3.7 \AA$ ). A rescaled and offset spectrum of 9P/Tempel 1, obtained by Meech et al. (2011), is overlaid to illustrate the locations of the $\mathrm{OH}(0-0)$ and $\mathrm{CN}(0-0)$ emission bands.

uncertainty, with the neutral slopes found at shorter wavelengths $(2 \pm 2 \% / 1000 \AA)$, with no evidence for absorption features due to ice or minerals. Again there is a contrast with typical comets, where the spectral slope changes between the visible and NIR (e.g. Snodgrass et al. 2016).

\subsection{Search for gas emission}

Figure 2 shows the combined, flux calibrated, spectrum (without removing the dust continuum) in the region where strong cometary emission bands are expected. We do not detect any emission above the noise level. The fluorescence scattering efficiency ( $g$-factor) of $\mathrm{OH}$ is a strong function of heliocentric velocity due to the complex solar spectrum in this region. Using the heliocentric radial velocity of $\mathrm{P} / 2010 \mathrm{~T} 1$ of $+1.9 \mathrm{~km} \mathrm{~s}^{-1}$ at this time and the corresponding $g$-factor calculated by Schleicher \& A'Hearn (1988) of $g[\mathrm{OH}(0-0)]=1.74 \times$ $10^{-15} r^{-2} \mathrm{erg} \mathrm{s}^{-1}$ molecule ${ }^{-1}$, our upper limit on the flux corresponds to a total number of $\mathrm{OH}$ molecules within the $\mathrm{X}$-shooter slit of $N(\mathrm{OH})<4.8 \times 10^{27}$.

To derive the corresponding upper limit to the sublimation rate requires the use of a coma density model. To facilitate comparison with other comet studies, we first use the common Haser model (Haser 1957). We adopt the parent and daughter scalelengths used by A'Hearn et al. (1995) and a nominal parent velocity given by $v_{\mathrm{p}}=0.85 r^{-0.5}=0.6 \mathrm{~km} \mathrm{~s}^{-1}$ (Cochran \& Schleicher 1993). Numerically integrating the resulting theoretical column densities within our slit implies a total production rate of $\mathrm{OH}$ of $Q(\mathrm{OH})<6 \times 10^{25}$ molecules $\mathrm{s}^{-1}$. As our observations occurred near solar maximum we use the active Sun branching ratio of 0.80 (Huebner et al. 1992), giving a production rate of $Q\left(\mathrm{H}_{2} \mathrm{O}\right)<8 \times 10^{25}$ molecules s ${ }^{-1}$ from the Haser model.

Although this result is directly comparable with many other analyses, the analytical Haser model contains several unphysical assumptions. Therefore we have also calculated an upper limit to $Q\left(\mathrm{H}_{2} \mathrm{O}\right)$ using a version of the Monte Carlo model first described by Combi \& Delsemme (1980). We use a Maxwellian velocity distribution for the parent $\mathrm{H}_{2} \mathrm{O}$ molecules, and we assume ejection only from the sunward hemisphere with a distribution proportional to $\cos \theta$, where $\theta$ is the subsolar latitude. 
The true photodissociation lifetimes of $\mathrm{H}_{2} \mathrm{O}$ and $\mathrm{OH}$ are dependent on the variable FUV and solar $\mathrm{H}-\alpha$ flux. From solar indices measured during our observations and the relationships given by Budzien et al. (1994), we use lifetimes against dissociation at 1 AU of $\tau\left(\mathrm{H}_{2} \mathrm{O}\right)=8.9 \times 10^{4} \mathrm{~s}$ and $\tau(\mathrm{OH})=1.5 \times 10^{5} \mathrm{~s}$. An analysis of previous ground-based measurements of outflow velocities was performed by Tseng et al. (2007), but these were all for comets with $Q\left(\mathrm{H}_{2} \mathrm{O}\right) \geq 10^{28}$ molecules s $^{-1}$. Instead we utilise the measurements made by the MIRO instrument onboard Rosetta when it arrived at 67P in August 2014 and the nucleus was in a low-activity state. Lee et al. (2015) found a clear correlation between the terminal expansion velocity and production rate of $\mathrm{H}_{2} \mathrm{O}$, with $v_{\mathrm{p}} \simeq 0.73 \mathrm{~km} \mathrm{~s}^{-1}$ for production rates of $Q\left(\mathrm{H}_{2} \mathrm{O}\right) \geq 10^{25}$ molecules s${ }^{-1}$ when the comet was at $r \simeq 3.5 \mathrm{AU}$. Assuming a $v_{\mathrm{p}} \propto r^{-0.5}$ relationship as expected from sublimation theory, we therefore assume $v_{\mathrm{p}}=0.8 \mathrm{~km} \mathrm{~s}^{-1}$ for $\mathrm{P} / 2012 \mathrm{~T} 1$ at the time of our observations. With these parameters and again using a branching ratio of 0.8 , we obtain $Q\left(\mathrm{H}_{2} \mathrm{O}\right)<9 \times 10^{25}$ molecules s ${ }^{-1}$.

We have performed similar modelling for our upper limit to the $\mathrm{CN} \Delta v=0$ flux. With a $g$-factor of $3.12 \times$ $10^{-13} r^{-2} \mathrm{erg} \mathrm{s}^{-1}$ molecule ${ }^{-1}$ (Schleicher 2010), the number of $\mathrm{CN}$ molecules in our slit is $N(\mathrm{CN})<4.0 \times 10^{24}$. Haser modelling then gives a production rate of $Q(\mathrm{HCN})<3 \times$ $10^{22}$ molecules s ${ }^{-1}$, assuming all $\mathrm{CN}$ is a photodissociation product of HCN. For the Monte Carlo model we use the same outflow velocity as before of $0.8 \mathrm{~km} \mathrm{~s}^{-1}$ and photodissociation lifetimes from Huebner et al. (1992) for an active sun of $\tau(\mathrm{HCN})=3.2 \times 10^{4} r^{2} \mathrm{~s}$ and $\tau(\mathrm{CN})=1.35 \times 10^{5} r^{2} \mathrm{~s}$. Using these parameters resulted in the same upper limit of $Q(\mathrm{HCN})<$ $3 \times 10^{22}$ molecules s ${ }^{-1}$.

\section{Discussion}

\subsection{Comparison with previous searches for gas emission}

There are two previously published attempts to detect gas emission from $\mathrm{P} / 2012 \mathrm{~T} 1$ : a spectrum covering the $\mathrm{CN}$ region using the Keck telescope (Hsieh et al. 2013), and a search for water using the ESA Herschel space telescope (O'Rourke et al. 2013). Neither attempt was successful. Hsieh et al. (2013) derived an upper limit of $Q(\mathrm{CN})<1.5 \times 10^{23}$ molecules s $^{-1}$ using a Haser model. Assuming a ratio $Q(\mathrm{CN}) / Q(\mathrm{OH})=3 \times 10^{-3}$ as found in normal comets (A'Hearn et al. 1995) and a branching ratio of 0.9 this corresponded to $Q\left(\mathrm{H}_{2} \mathrm{O}\right)<5 \times 10^{25}$ molecules s $^{-1}$. Our improved limit on $\mathrm{CN}$ production from our X-shooter observations, assuming the same composition, implies a water production limit of $Q\left(\mathrm{H}_{2} \mathrm{O}\right)<1 \times 10^{25}$ molecules $\mathrm{s}^{-1}$. This improvement is largely due to the significantly longer total integration time used at the VLT compared to the Keck observations, and $\mathrm{UVB}$ arm of $\mathrm{X}$-shooter being very efficient around the $\mathrm{CN}$ band.

The Herschel observation has the unique advantage of searching directly for water, and found a limit of $Q\left(\mathrm{H}_{2} \mathrm{O}\right)<$ $7.63 \times 10^{25}$ molecules s ${ }^{-1}$ (O'Rourke et al. 2013). Although higher than the result from $\mathrm{CN}$, there are less assumptions involved in this upper limit and we therefore regard it as the best previous limit on water production from a MBC. The limit on water production derived from our X-shooter search for $\mathrm{OH}$ emissions is $Q\left(\mathrm{H}_{2} \mathrm{O}\right)<9 \times 10^{25}$ molecules $\mathrm{s}^{-1}$, similar to the Herschel value. Additionally, the Herschel observations took place approximately one month after our VLT observations, due to pointing constraints to keep the telescope behind its sunshade, where according to the lightcurve of Hsieh et al. (2013) activity had decreased since the epoch of our observations.

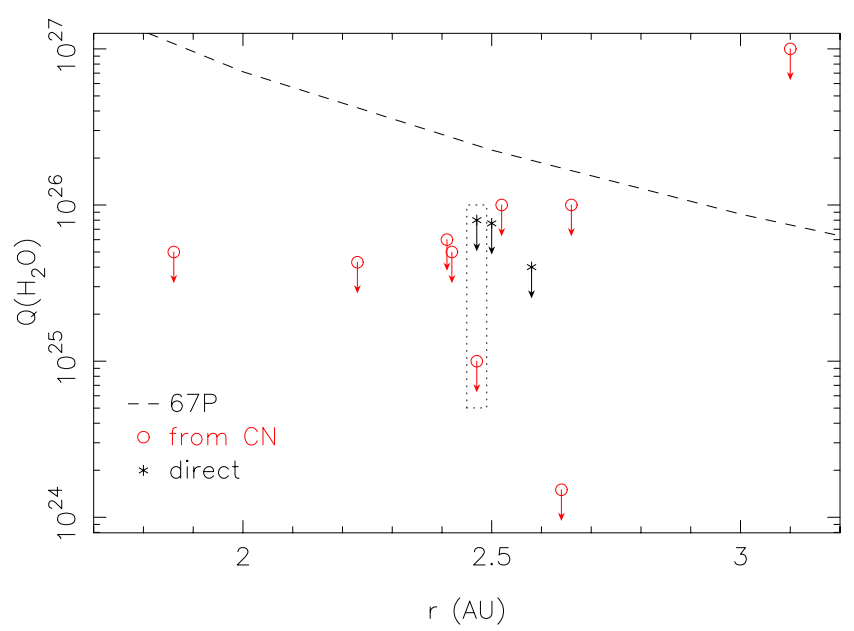

Fig. 3. Upper limits on water production, via $\mathrm{CN}$ or direct $\left(\mathrm{H}_{2} \mathrm{O}\right.$ or $\left.\mathrm{OH}\right)$. Water production rate for 67P, as a typical JFC, shown by dashed line for comparison (empirical fit to inbound data - Hansen et al. 2016). Our $\mathrm{X}$-shooter results are highlighted by the dotted box.

In Fig. 3 we show these various upper limits, along with the other attempts for MBCs listed in Table 1. It is clear that all upper limits fall around the same production rate, with a weak trend towards more distant objects having higher (less constraining) limits due to their relative faintness and the $g$-factor's $g \propto r^{-2}$ dependence. The most constraining limits come from our X-shooter $\mathrm{CN}$ measurement, which had a longer integration time, and the limit for 133P published by Licandro et al. (2011), which was derived from a column density through the outer coma region and a vectorial model, and therefore may not be directly comparable. This plot largely shows the limit of sensitivity of the current generation of large telescopes.

Figure 3 also shows the water production rate for $67 \mathrm{P}$ as a "typical" JFC for comparison, which was higher at main belt distances, demonstrating that these upper limits at least show MBCs to be very weakly active comets. However, it is worth noting that attempts to detect $\mathrm{CN}$ in 67P from the ground at large $r$ inbound also produced only upper limits, with a similar sensitivity to the MBC searches (Snodgrass et al. 2016): the comparison between ground-based observations and Rosetta measurements shows that the $\mathrm{CN} / \mathrm{H}_{2} \mathrm{O}$ ratio varies significantly, even within one apparition for a single comet.

\subsection{Comparison with dust activity}

As there are no reported detections of gas associated with MBCs to date, we use the observed dust coma to estimate the possible sublimation rate from $\mathrm{P} / 2012 \mathrm{~T} 1$. Hsieh et al. (2013) report an absolute $R$-band magnitude of $m_{R}(1,1,0) \simeq 15.5$ within a 5 arcsec radius aperture near the epoch of our observations, equivalent to a comet dust activity index of $A f \rho \simeq 10 \mathrm{~cm}$. Moreno et al. (2013) measured a fainter $m_{R}(1,1,0) \simeq 17.3$ with a smaller aperture, however as they do not report the aperture size we do not use their data. Assuming a typical cometary dust particle albedo of $A=0.04$ gives a total dust cross section within the aperture of $6 \times 10^{7} \mathrm{~cm}^{2}$. Taking a mean particle diameter of $\sim 10^{-6} \mathrm{~m}$ and assuming a dust outflow velocity slower than the gas of $\sim 0.1 \mathrm{~km} \mathrm{~s}^{-1}$ implies a dust production rate of $\sim 7 \times 10^{19}$ particles $\mathrm{s}^{-1}$. With an assumed density of $1000 \mathrm{~kg} \mathrm{~m}^{-3}$ this gives a mass loss rate in dust at this time of $\sim 0.6 \mathrm{~kg} \mathrm{~s}^{-1}$.

Using sophisticated modelling of the dust coma and tail, Moreno et al. (2013) calculate a dust mass loss rate at the time 
of our observations $\sim 90$ days after perihelion of $\sim 0.2-0.3 \mathrm{~kg} \mathrm{~s}^{-1}$, within a factor $2-3$ of the simplistic calculation above. As $\mathrm{P} / 2012$ $\mathrm{T} 1$ was observed near perihelion, we assume that $\mathrm{H}_{2} \mathrm{O}$ sublimation was the main driver of activity. The estimated gas/dust mass loss ratio varies significantly between normal comets. If we assume a nominal ratio of $\sim 1$ for $\mathrm{P} / 2012 \mathrm{~T} 1$, this gives a water sublimation rate of $Q\left(\mathrm{H}_{2} \mathrm{O}\right) \sim 1-2 \times 10^{25}$ molecules s $^{-1}$. This is below both our upper limit of $Q\left(\mathrm{H}_{2} \mathrm{O}\right)$ and that of O'Rourke et al. (2013), but by less than a factor of 10 . This strongly indicates that direct detection of $\mathrm{OH}$ at future perihelion passages may be possible if P/2012 T1 exhibits the same level of activity. Importantly, we also note that this rules out a very high gas/dust ratio of $\geq 10$ for this MBC. It is possible that $\mathrm{P} / 2012 \mathrm{~T} 1$ has a gas/dust ratio $<1$, as was observed by Rosetta at 67P (Rotundi et al. 2015), which would imply a true gas production further below our derived limit.

Assuming the normal $Q(\mathrm{CN}) / Q(\mathrm{OH})$ and branching ratios, our upper limit for water production based on our $\mathrm{CN}$ upper limit would be $Q\left(\mathrm{H}_{2} \mathrm{O}\right) \sim 1 \times 10^{25}$ molecules $\mathrm{s}^{-1}$. This is of the same order as the dust mass-loss rate. As we do not detect $\mathrm{CN}$, we are led to conclude that this object has a similar or lower $Q(\mathrm{CN}) / Q\left(\mathrm{H}_{2} \mathrm{O}\right)$ than normal comets, or that $Q($ gas $) / Q($ dust $)<1$, or some combination of both. While this potentially supports the models of Prialnik \& Rosenberg (2009) as discussed above, it is clear that significantly deeper integrations are required to further investigate this.

From our upper limit to $Q(\mathrm{OH})$, we can use standard sublimation theory to estimate an upper limit to the effective actively sublimating area. We assume a C-type taxonomy with a geometric albedo of 0.06 (Mainzer et al. 2011) and a phase integral of 0.3 , giving a Bond albedo for the surface of $\sim 0.018$. Taking the vapour pressure and latent heats for $\mathrm{H}_{2} \mathrm{O}$ from Prialnik et al. (2004), bare ice would sublimate at a rate of $1.8 \times 10^{21}$ molecules s ${ }^{-1} \mathrm{~m}^{-2}$. Therefore our non-detection of $\mathrm{OH}$, the upper limit of $Q\left(\mathrm{H}_{2} \mathrm{O}\right)<9 \times 10^{25}$ molecules s $^{-1}$ corresponds to a bare ice patch of area $\leq 5 \times 10^{4} \mathrm{~m}^{2}$. This in turn corresponds to $0.2 \%$ of surface area if the nucleus radius is $1.3 \mathrm{~km}$, which is the upper limit from photometry taken when the comet was active (O'Rourke et al. 2013). The active patch could be as much as $4 \%$ of the surface if the true size of the nucleus is as small as smallest known MBC, 259P/Garradd (radius $=300 \mathrm{~m}$; MacLennan \& Hsieh 2012). These active fraction areas are comparable with those found for typical comets (A'Hearn et al. 1995).

\section{Conclusions}

Unfortunately, all of the available upper limits are consistent with the predicted production rates required to drive the observed dust production, and are therefore not constraining on theory water ice sublimation remains the most likely explanation for the repeated activity of MBCs, but at a level below current detection limits. $\mathrm{X}$-shooter limits on water production via $\mathrm{OH}$ are similar to previous results using $\mathrm{CN}$ as a proxy, but have the advantage of not relying on an unknown $\mathrm{CN} / \mathrm{H}_{2} \mathrm{O}$ ratio for MBCs. Our upper limits may be within one order of magnitude of detecting outgassing, based on some reasonable assumptions, but we will require a brighter $\mathrm{MBC}$ to make the first direct gas detection with current technology. In a few years, the launch of the James Webb Space Telescope will give a powerful direct way to study water outgassing from comets; it is expected to be sensitive enough to detect the water escaping from MBCs (Kelley et al. 2016).

Acknowledgements. We thank the ESO DG and DDT panel for awarding time to this project, and the service mode observer, telescope operator and USD staff who enabled us to get the data. We thank Dan Bramich for useful advice on the X-shooter pipeline. C.S. received funding from the European Union Seventh Framework Programme (FP7/2007-2013) under grant agreement No. 268421 and from the UK STFC in the form of an Ernest Rutherford Fellowship. A.F. acknowledges support from UK STFC grant ST/L000709/1. This paper was written, in part, at the International Space Science Institute (ISSI) in Bern, Switzerland. We thank ISSI for their support.

\section{References}

A'Hearn, M. F., Millis, R. L., Schleicher, D. G., Osip, D. J., \& Birch, P. V. 1995, Icarus, 118, 223

Bockelée-Morvan, D., Crovisier, J., Mumma, M. J., \& Weaver, H. A. 2004, in Comets II, eds. M. C. Festou, H. U. Keller, \& H. A. Weaver (University of Arizona Press), 391

Bodewits, D., Kelley, M. S., Li, J.-Y., et al. 2011, ApJ, 733, L3

Budzien, S. A., Festou, M. C., \& Feldman, P. D. 1994, Icarus, 107, 164

Capria, M. T., Marchi, S., de Sanctis, M. C., Coradini, A., \& Ammannito, E. 2012, A\&A, 537, A71

Cochran, A. L., \& Schleicher, D. G. 1993, Icarus, 105, 235

Combi, M. R., \& Delsemme, A. H. 1980, ApJ, 237, 633

de Val-Borro, M., Rezac, L., Hartogh, P., et al. 2012, A\&A, 546, L4

Feldman, P. D., Cochran, A. L., \& Combi, M. R. 2004, in Comets II, eds. M. C.

Festou, H. U. Keller, \& H. A. Weaver (University of Arizona Press), 425

Hadamcik, E., \& Levasseur-Regourd, A. C. 2009, Planet. Space Sci., 57, 1118

Hainaut, O. R., Boehnhardt, H., Snodgrass, C., et al. 2014, A\&A, 563, A75

Hansen, K. C., Altwegg, K., Berthelier, J.-J., et al. 2016, MNRAS, 462, S491

Haser, L. 1957, Bulletin de la Société Royale des Sciences de Liège, 43, 740

Hsieh, H. H., \& Jewitt, D. C. 2006, Science, 312, 561

Hsieh, H. H., Jewitt, D., Lacerda, P., Lowry, S. C., \& Snodgrass, C. 2010, MNRAS, 403, 363

Hsieh, H. H., Meech, K. J., \& Pittichová, J. 2011, ApJ, 736, L18

Hsieh, H. H., Yang, B., \& Haghighipour, N. 2012a, ApJ, 744, 9

Hsieh, H. H., Yang, B., Haghighipour, N., et al. 2012b, ApJ, 748, L15

Hsieh, H. H., Yang, B., Haghighipour, N., et al. 2012c, AJ, 143, 104

Hsieh, H. H., Kaluna, H. M., Novaković, B., et al. 2013, ApJ, 771, L1

Hsieh, H. H., Denneau, L., Fitzsimmons, A., et al. 2014, AJ, 147, 89

Huebner, W. F., Keady, J. J., \& Lyon, S. P. 1992, Ap\&SS, 195, 1

Ishiguro, M., Hanayama, H., Hasegawa, S., et al. 2011, ApJ, 740, L11

Jewitt, D. 2012, AJ, 143, 66

Jewitt, D., Yang, B., \& Haghighipour, N. 2009, AJ, 137, 4313

Jewitt, D., Agarwal, J., Weaver, H., Mutchler, M., \& Larson, S. 2013, ApJ, 778, L21

Jewitt, D., Agarwal, J., Li, J., et al. 2014, ApJ, 784, L8

Jewitt, D., Agarwal, J., Peixinho, N., et al. 2015, AJ, 149, 81

Kelley, M. S. P., Woodward, C. E., Bodewits, D., et al. 2016, PASP, 128, 018009 Kolokolova, L., Hanner, M. S., Levasseur-Regourd, A.-C., \& Gustafson, B. Å. S.

2004, in Comets II, eds. M. C. Festou, H. U. Keller, \& H. A. Weaver (University of Arizona Press), 577

Lee, S., von Allmen, P., Allen, M., et al. 2015, A\&A, 583, A5

Licandro, J., Campins, H., Tozzi, G. P., et al. 2011, A\&A, 532, A65

Licandro, J., Moreno, F., de León, J., et al. 2013, A\&A, 550, A17

MacLennan, E. M., \& Hsieh, H. H. 2012, ApJ, 758, L3

Mainzer, A., Grav, T., Masiero, J., et al. 2011, ApJ, 741, 90

Meech, K. J., Pittichová, J., Yang, B., et al. 2011, Icarus, 213, 323

Modigliani, A., Goldoni, P., Royer, F., et al. 2010, in SPIE Conf. Ser., 7737, eds. D. R. Silva, A. B. Peck, \& B. T. Soifer, 773728

Moreno, F., Cabrera-Lavers, A., Vaduvescu, O., Licandro, J., \& Pozuelos, F. 2013, ApJ, 770, L30

O’Rourke, L., Snodgrass, C., de Val-Borro, M., et al. 2013, ApJ, 774, L13

Prialnik, D., \& Rosenberg, E. D. 2009, MNRAS, 399, L79

Prialnik, D., Benkhoff, J., \& Podolak, M. 2004, in Comets II, eds. M. C. Festou,

H. U. Keller, \& H. A. Weaver (University of Arizona Press), 359

Rotundi, A., Sierks, H., Della Corte, V., et al. 2015, Science, 347, 3905

Schleicher, D. G. 2010, AJ, 140, 973

Schleicher, D. G., \& A'Hearn, M. F. 1988, ApJ, 331, 1058

Snodgrass, C., Tubiana, C., Vincent, J.-B., et al. 2010, Nature, 467, 814

Snodgrass, C., Jehin, E., Manfroid, J., et al. 2016, A\&A, 588, A80

Stevenson, R., Kramer, E. A., Bauer, J. M., Masiero, J. R., \& Mainzer, A. K. 2012, ApJ, 759, 142

Tholen, D. J., Elliott, T., Sato, H., et al. 2012, Central Bureau Electronic Telegrams, 3252, 1

Tseng, W.-L., Bockelée-Morvan, D., Crovisier, J., Colom, P., \& Ip, W.-H. 2007, A\&A, 467, 729

Vernet, J., Dekker, H., D’Odorico, S., et al. 2011, A\&A, 536, A105

Yang, B., \& Hsieh, H. 2011, ApJ, 737, L39 